\title{
Multi-photon ablation of biological samples with custom- built femtosecond fiber laser-microscope system
}

\author{
S. Yavaş ${ }^{1}$, M. Erdoğan ${ }^{1}$, K. Gürel ${ }^{2}$, U. H. Tazebay ${ }^{3}$, and F. Ö. Ilday ${ }^{2}$ \\ ${ }^{1}$ Materials Science and Nanotechnology Graduate Program, Bilkent University, TR-06800 Ankara, Turkey \\ ${ }^{2}$ Department of Physics, Bilkent University, TR-06800 Ankara, Turkey \\ ${ }^{3}$ Department of Molecular Biology and Genetics, Bilkent University, TR-06800 Ankara, Turkey
}

\begin{abstract}
A femtosecond laser-microscope system is custom-built for ablation of cells and tissue at $1030 \mathrm{~nm}$. Fiber lasers offer important advantages for nanosurgery, including superior robustness, lower-cost and nearly complete control over pulse train pattern.

(C)2010 Optical Society of America

OCIS codes: (170.7160) Ultrafast technology; (170.1020) Ablation of tissue
\end{abstract}

Ultrashort laser pulses are increasingly used in biological applications in recent years. In addition to their well-known use in nonlinear imaging [1], manipulation and dissection of individual cells in tissue [2] or structures inside living cells and other biological materials can be accomplished using femtosecond pulses with nanoscale precision [3,4]. To date, all of these experiments have been performed using solid state lasers, most commonly Ti:sapphire. While these lasers offer good technical performance, they are large in size, costly and very complex. The oscillators operate at a fixed repetition rate around $80 \mathrm{MHz}$ and amplifier-based systems are typically constrained to a few $\mathrm{kHz}$. Control of the repetition rate for an oscillator requires a complex and expensive Pockels cell. The necessary pulse durations and energies for these applications are within the range of femtosecond fiber lasers, which are more compact, simpler to operate, and cost much less. Importantly, the repetition rate and the pulse train can be controlled using acousto-optic modulators (AOM). Since the addition of an inline fiber amplifier following pulse picking is easy, any reduction in pulse can be compensated for. In addition, the intensity noise of fiber lasers can be made extremely small [5], which directly relates to the ablation precision directly.However, despite the clearly high potential, fiber lasers have not been utilized in this area.

Here, we report, for the first time to our knowledge, the use of an Yb-doped fiber laser custom-designed for the purpose of nanosurgery and related applications, delivering femtosecond pulses at controllable repetition rates.

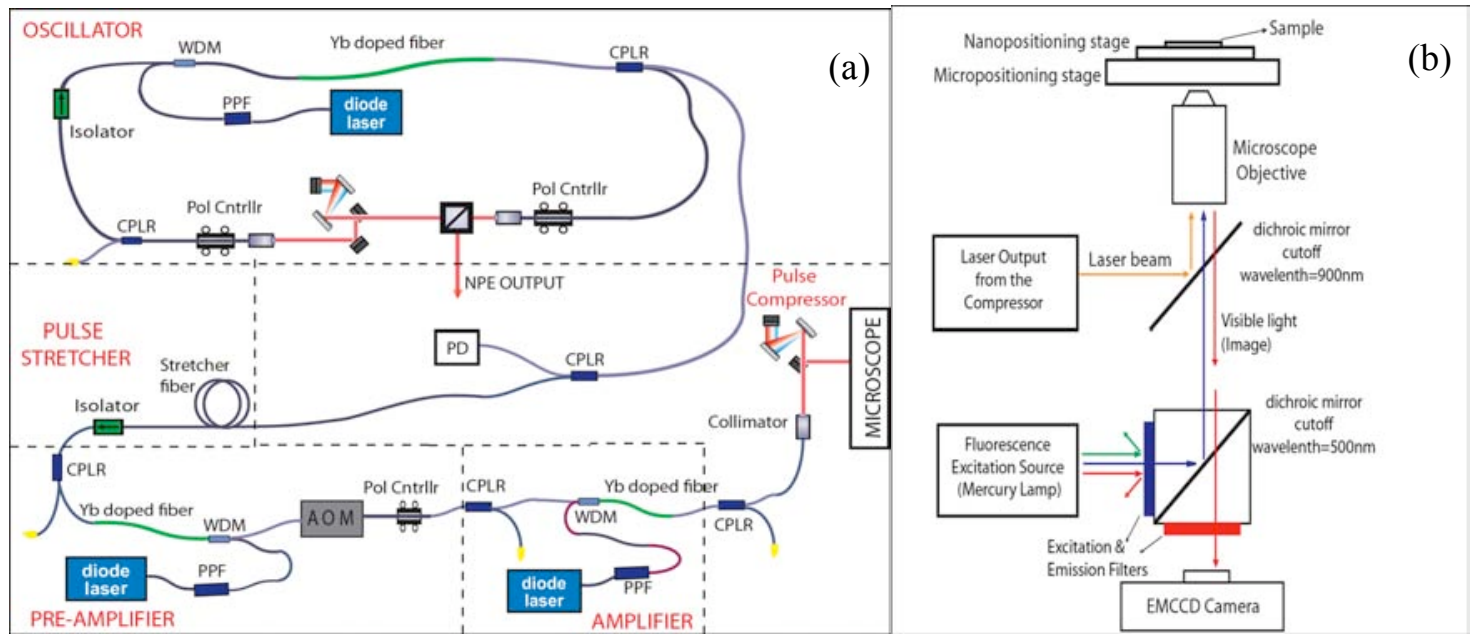

Fig. 1. (a) Schematic of the Yb-doped fiber laser-amplifier setup. WDM: wavelength division multiplexing; CPLR: fiber coupler; PD: Photo Diode; AOM: acousto-optic modulator; PPF: pump-protection filter. (b)Schematic of the femtosecond laser-fluorescence microscope optics.

The experimental setup is shown schematically in Fig. 1(a). The seed oscillator is a stretched-pulse Yb-doped fiber laser[6]. It produces chirped pulses at $32 \mathrm{MHz}$ of repetition rateat $1030 \mathrm{~nm}$. The output is directly coupled to the pulse stretcher-preamplifier pair, which stretches the pulses to $\sim 20 \mathrm{ps}$ in a $20 \mathrm{~m}$-long fiber and amplifies to $3 \mathrm{~nJ}$. After the pre-amplifier, the pulses traverse a fiber-integrated AOM to reduce the repetition rate, nominally, to 4 MHz. The pulses are then amplified to $60 \mathrm{~nJ}$. Pulses are compressed to $170 \mathrm{fs}$ in a grating pair. A second, freespace AOM allows gating of the individual pulses. A telescope is used to expand the beam to completely fill the back aperture of the objective. The pulse picking electronics have been developed in-house and are controlled by an FPGA board, addressable with a PC. This affords us nearly complete flexibility in pulse picking.

Inside the customized epi-fluorescent microscope structure, laser beam is directed to the objective with a dichroic mirror housed in an extra turret. The dichroic mirror is highly reflective at the laser wavelength, and 
transmissive for visible light and the fluorescence excitation (Fig. 1(b)). Sampling positioning is accomplished with a step-motor based 2-D micropositioning stage and 3-D piezo stage for precise with $\sim 20 \mathrm{~nm}$ precision. Visualizationis based on with fluorescent and phase-contrast imaging.A 60X,1.2-NA objective (Nikon CFI Plan Apochromat VC 60X, water immersion) is used for sub-cellular ablation and imaging and a 20X, 0.4-NA, phasecontrast objective (Nikon CFI S Plan Fluor ELWD ADM 20X) is used for multicellular/tissue-level ablation and imaging. A high-sensitivity EMCCD camera is used for imaging. All major aspects of the laser-microscope system are accomplished via a PC. Control of sample position with a computer joystick allows ease of use.
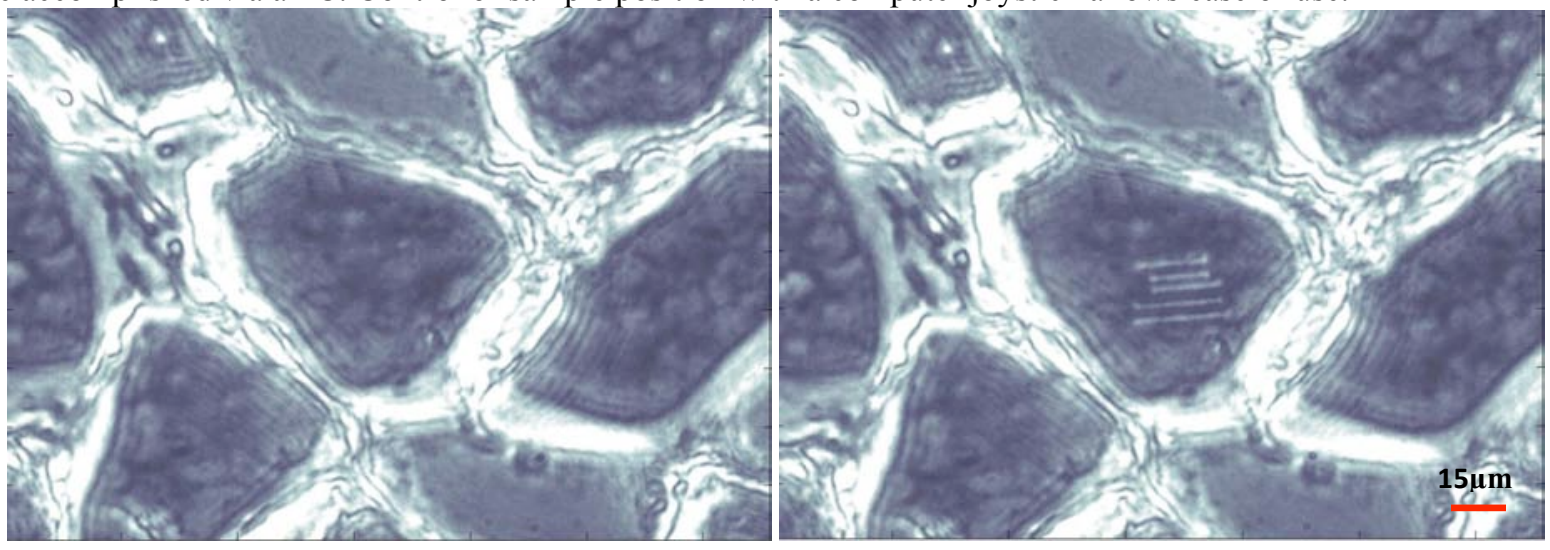

Fig. 2. Tissue sample (mouse gastrocnemius muscle section)before and after ablation with the femtosecond fiber laser.
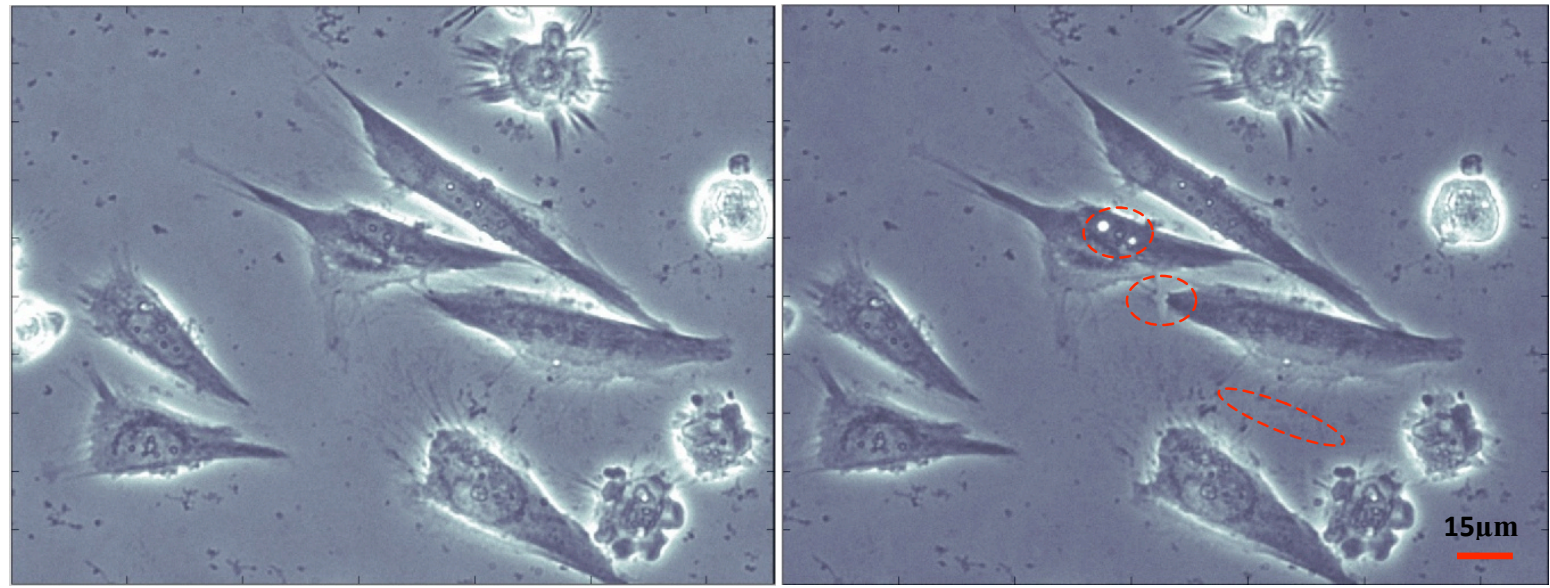

Fig. 3. Sub-cellular surgery on fixed Saos-2 cells: (a) before and (b) after ablation with the femtosecond fiber laser.

As a demonstration of the system's capability, 170-fs, 7-nJ pulses at $4 \mathrm{MHz}$ are used to make cuts on the section of mouse gastrocnemius muscle. The beam is focused onto the surface with a spot size of approximately $4 \mu \mathrm{m}$ using the $20 \mathrm{X}$ objective. 5 parallel, linear cuts are made, as shown in Fig. 2.The line widths are measured to be $2-2.5 \mu \mathrm{m}$, consistent with the spot size of the objective. Next, sub-cellular surgery is performed on Saos-2 cells using the $60 \mathrm{X}$ objective. In this mode of operation, sub-micron features are obtained (Fig. 3), which is beyond the diffraction limit and confirms the multi-photon nature of the ablation process.

In conclusion, we have demonstrated a custom-built laser-microscope system for nanosurgery and tissue ablation experiments. This is the first time, to our knowledge, that a fiber laser-amplifier system, using standard optical components only, is used in this application. Custom-design and the use of fiber architecture allows increased flexibility, including virtually complete control over pulse picking, while keeping individual pulse energy and pulse duration the same. Given the increased flexibility, ease-of-use and lower-cost of the fiber laserbased system described here, we expect that the fiber laser-based system will find numerous applications in nanosurgery and tissue ablation.

[1] W. Denk, J.H. Strickler, and W.W. Webb, “Two-photon laser scanning fluorescence microscopy,” Science 248, 4951, 73 (1990).

[2] P.T.C. So, H. Kim, and I.E. Kochevar, "Two-photon deep tissue ex vivo imaging of mouse dermal andsubcutaneous structures," Opt. Express 3, 339 (1998).

[3] A. Vogel, J. Noack, G. Huttman, G. Paltauf, "Mechanisms of femtosecond laser nanosurgery of cells and tissues," Appl. Phys. B 81, 1015 (2005).

[4] S. H. Chung, E. Mazur, "Surgical applications of femtosecond lasers,"J. Biophoton. 2, No. 10, 557-572 (2009)

[5] İ. L. Budunoğlu, C. Ülgüdür, B.Oktem, and F. Ö. Ilday, "Intensity noise of mode-locked fiber lasers", Opt. Lett. 34, 2516 (2009).

[6] H. Lim, F. Ö. Ilday, and F. W. Wise, “Generation of 2-nJ pulses from a femtosecond Yb fiber laser”, Opt. Lett. 28, 660 (2003). 